\title{
Fill My Datebook: A software tool to generate and handle lists of events
}

\author{
LARS LEWEJOHANN \\ University of Münster, Münster, Germany
}

\begin{abstract}
Electronic calendars, and especially Internet-based calendars, are becoming more and more popular. Their advantages over paper calendars include being able to easily share events with others, gain remote access, organize multiple calendars, and receive visible and audible reminders. Scientific experiments often include a huge number of events that have to be organized. Experimental schedules that follow a fixed scheme can be described as lists of events. The software application presented here allows for the easy generation, management, and storage of lists of events using the Internet-based application Google Calendar.
\end{abstract}

Behavioral experiments usually include a number of test subjects and often a set of different measures to be taken with each individual. The measures taken usually follow a fixed timing schedule synchronized to certain characteristics of the subjects (e.g., the first measure taken might be date of birth). If the schedule comprises more than a single day, the organization of events naturally requires a calendar. The scheduling of such events is often iterative and requires a great amount of coordination. If this is done in a paper calendar, usually a considerable amount of erasing and crossing out occurs because of rescheduling. Although electronic calendars outperform paper calendars in almost all respects, acceptance of these tools is not yet fully established. In a family survey (Hutchinson, Bederson, Plaisant, \& Druin, 2002), the authors stated that even heavy computer users still rely on paper calendars. Merely out of habit, many researchers spend a considerable amount of time filling in large paper calendars. Making people change their calendaring habits therefore must include a benefit in time savings, which can occur only when the learning curve for the electronic calendar is not too steep and the number of entries required exceeds an individually given limit. For example, in a research study on behavioral phenotyping of knockout mice, a battery of tests was applied (Lewejohann et al., 2004). In this study, mice were tested at certain ages in different behavioral paradigms. The study incorporated 170 mice and 11 different tests that were carried out with each mouse. For each mouse, a list of events based on the date of birth was generated. Although many individuals could be grouped because they shared the same date of birth, this experimental setup led to a huge number of events that would have been laborious to manually enter into a calendar. In particular, the calculation of dates on the basis of an offset between events cannot be solved by simple math, especially when the offset spans a period of months. Besides manually counting day by day, the resulting dates could be calculated by using date-formatted cells in spreadsheet software. This is likely the most commonly used approach for the organization of lists of events. In the above-mentioned study, the dates were organized using proprietary software on a Palm handheld computer. In recent years, this software has been modified from time to time, and new versions were compiled for different experimental designs. However, acceptance of handheld computers was low among students, mainly because of difficulties with the pen-based data input, which required specific lettering for handwriting recognition. Therefore, a new version of the software had to be made using a more widely accepted type of electronic calendar. Various online calendars are freely available through the World Wide Web. One key feature of online calendars is the possibility of collective use of individual calendars between multiple users, which is crucial for allowing coordination benefits (van den Hooff, 2004). Therefore, using an online calendar for the organization of experimental schedules is particularly suitable when more than one researcher is involved, since such calendars can be shared by different users and can be accessed worldwide, from any working Internet connection. In addition, sharing calendars with other researchers from the same laboratory may help to avoid conflicting allocations of the facilities used for experiments.

\section{Google Calendar}

When Google introduced its calendar service in 2006, acceptance of online calendars received a fresh impetus, with a rapidly increasing number of users worldwide. Events can easily be viewed, added, or deleted online with this service, and even drag-and-drop of events from one date to another is supported. The application allows for the creation and viewing of multiple calendars in the same

L. Lewejohann, ljohann@phenotyping.com 


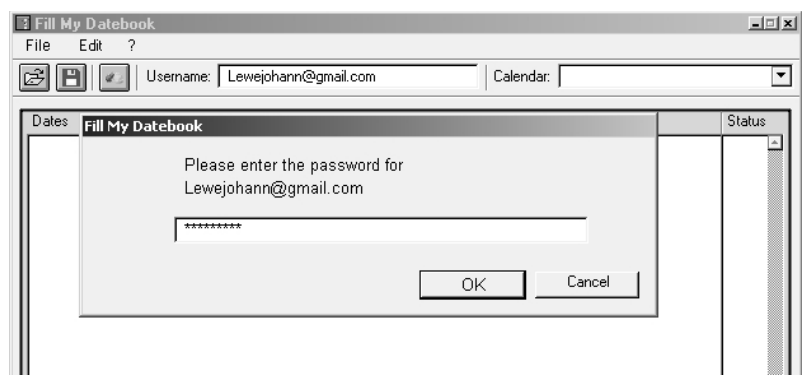

Figure 1. The user enters a username into Google Calendar, and after submitting the password, a secure connection is established for access to the user's calendar(s).

view, using different colors for each calendar. Calendars can also be shared, with different levels of access for different users. Event reminders can be configured to be sent via e-mail, as well as by text messaging to mobile phones. Data entered into the calendar are stored online, providing a reasonable level of security from hard drive failure. Google Calendar is written in Ajax ("Asynchronous JavaScript and XML"; see Garrett, 2005), which allows fast and user-friendly access to the calendar, and because it supports the iCalendar standard (tools.ietf.org/html/ rfc2445), it can exchange data with many other electronic calendars. Google Calendar allows other programs to view and generate calendar events through an application programming interface that is described in detail at a Web site provided by Google (code.google.com/apis/calendar/ overview.html). The calendar service is free, and the only prerequisite is registration of a Google account. Setting up a new calendar is very intuitive, and an elaborate manual covering all features of the calendar is available at www .google.com/support/calendar/.

It is recommended that a new calendar be initiated for each experimental design, in order to manage different experiments separately.

\section{Fill My Datebook}

The Fill My Datebook (FMDB) software discussed here can generate lists of events that can easily be edited in order to iteratively enter the same set of events for different individuals with different starting dates into a given calendar. FMDB will run on Microsoft Windows 32-bit systems. It requires an established Internet connection with the firewall configured to allow access to FMDB.exe itself and to curl.exe. FMDB establishes a secure communication according to the HTTPS protocol with the help of the cURL software (curl.haxx.se), which supports SSL (secure sockets layer) certificates. A current version of cURL is included in the installation package of FMDB. At its first startup, FMDB asks the user to enter the folder in which cURL will be installed. Usually, this will be the same folder FMDB is located in; however, if cURL is already installed on the computer, no secondary copy is needed. The e-mail address of the Google account has to be entered in the field labeled "Username" (see Figure 1). In order to establish a connection, at least one Google calendar has to be set up first.

The path to cURL and the username are saved for future use in a file named FMDB.ini, which can be edited with standard text editor software (e.g., pfe or Notepad ++ ). When the secure connection to the Google server is established, a list of calendars available for the specific user account is shown, and the desired calendar can be chosen.

The generation of a list of events is very simple. Suppose that a battery of tests is applied to a number of mice: All mice will undergo a general health check at 30 days of age and will be weighed at 60,90, and 120 days of age. At Day 125, they will be tested for locomotory behavior in an open-field test. An elevated plus-maze test will be conducted to test anxiety-related behavior at Day 130, and finally, from Days 140-145, the mice will be tested for spatial memory performance in a Barnes maze. This list results in a total of 11 dates for each individual mouse. Each event is scheduled according to the date of birth of the individual plus a given offset of days. In FMDB, you can pick the date of birth from a small calendar window and define the offset of days. For each event, you can enter a prefix, a title, a description, and a location. If all desired information is filled in, the event can be added to the list by pressing a button. In the example above, the first entry could look like the one presented in Table 1.

The date resulting from adding the offset to the date of birth (2007-05-29, in the example) is listed in FMDB, including the weekday ("Tu"). After the complete list is generated, it can be entered into Google Calendar by pressing the "Enter into calendar" button (see Figure 2).

The list of events can be saved for future use. If the same schedule is to be applied for a different subject (e.g., female1, born 2007-05-06), it is sufficient merely to change the start date, write "female1" in the Prefix field, and empty all other fields. Then, by pressing the "Update list with these values" button, the necessary changes are made in the list, which can subsequently be entered into the calendar. It might be of some value to avoid weekends and public holidays in experimental schedules. FMDB shows the resulting dates and weekdays calculated according to the given offset. Of course, all dates can be immediately rescheduled by changing the starting day. Single events can be moved by selecting the event in the list and changing the offset with the arrow keys of the keyboard. After events are entered into the calendar, they can still be moved within the

Table 1

Example of the Different Fields That Can Be Filled In (or Left Blank) for Each Date Entry in FMDB

\begin{tabular}{cccccc}
\hline Birth Date & Offset & Prefix & Title & Description & Location \\
\hline $2007-04-29$ & 30 & male1 & health check & reflexes, sensory, \& motor functions & room 123 \\
\hline
\end{tabular}




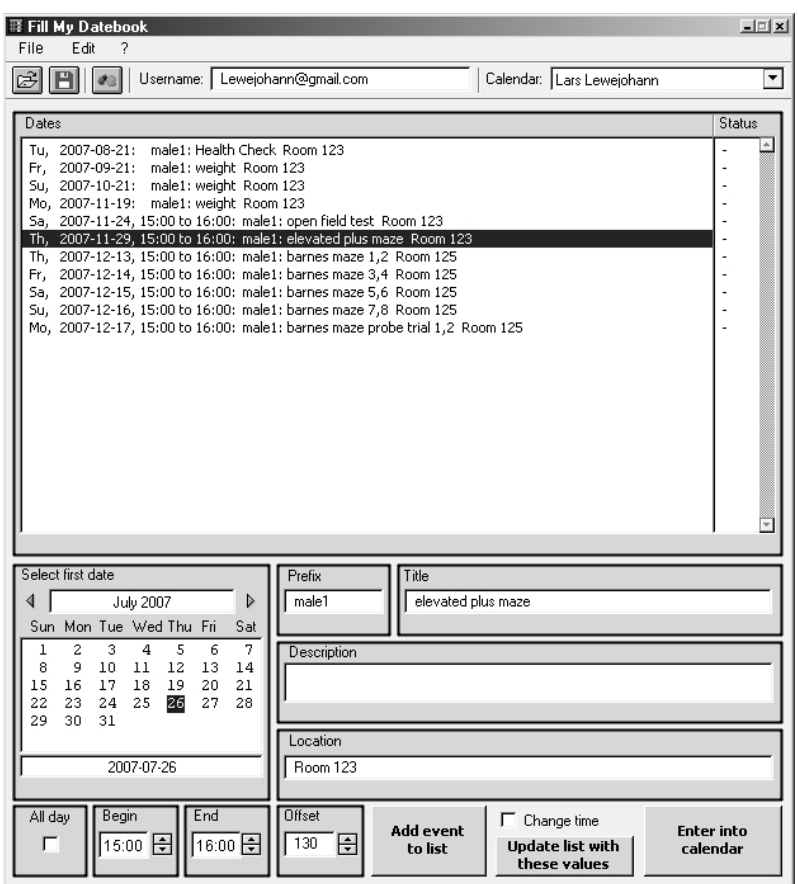

Figure 2. As a list of events is generated, the resulting dates and weekdays are shown. The complete list can be entered into Google Calendar by pressing the button at the lower right. By means of filling in a new start date and a different value in one of the fields (e.g., "female2" instead of "male1" in the Prefix field), leaving all other fields blank, the complete list can be updated with new values.

calendar by "drag and drop." This can be useful for events that are not symmetrically distributed along the time line, which otherwise might lead to a clustering of events.

\section{Discussion}

Changing calendaring habits requires great care, since a replacement for an existing paper calendar has to come along with perceptible advantages and simplicity. Also, some people tend to distrust computerized solutions, and therefore reliability is a key. The proposed solution here relies on the server facilities of one of the biggest computer companies, which can thus reasonably be thought of as reliable in terms of data integrity. In addition, calendars can be exported to other electronic calendars through the iCalendar standard, or the resulting calendar sheets can be printed out as a paper backup. The key features of FMDB are the straightforwardness of its function - that is, calculating target dates on the basis of an initial date plus an offset - and the possibility of iteratively handling lists of events. Either within FMDB or subsequently in the online calendar, events can easily be moved to different dates to avoid holidays or weekends or to prevent the clustering of experimental dates. Multiple calendars for different experiments can be added and shared with other experiment- ers, allowing various levels of permissions for the users. This enables collaboration and sharing of schedules and is therefore also suitable for the management of smaller testing facilities. Given the advantages of online over paper calendars and the benefits in time savings due to the easy generation and posting of lists of events, FMDB will be a valuable tool in research and may change the calendaring habits of many researchers.

\section{Program}

FMDB is a program that runs on Microsoft Windows 32-bit systems. FMDB could possibly be ported to other operating systems as well, such as Linux or MacOS, since it is written using Revolution (www.runrev.com), a crossplatform programming environment. However, the secure communication with Google Calendar is established by using the command-line tool cURL (curl.haxx.se), which supports SSL certificates. Therefore, secure communication has to be established separately for each operating system. The source code of FMDB is available on request. FMDB provides a graphical user interface in which all necessary settings and parameters can be entered.

\section{Availability}

An FMDB executable file for Windows and a complete manual can be downloaded from this Web site: www .phenotyping.com/fmdb.html. Authors who use FMDB for their research are encouraged to cite this article.

\section{Conclusion}

FMDB is a program that simplifies the generation of date book entries for experimental designs. It can help calculate offsets that would be time consuming if done manually or with other tools.

\section{AUTHOR NOTE}

Correspondence related to this article may be sent to L. Lewejohann, University of Münster, Department of Behavioral Biology, Badestr. 13, 48149 Münster, Germany (e-mail: ljohann@phenotyping.com).

\section{REFERENCES}

Garrett, J. J. (2005). Ajax: A new approach to Web applications Retrieved September 17, 2007, from www.adaptivepath.com/ideas/ essays/archives/000385.php.

Hutchinson, H., Bederson, B. B., Plaisant, C., \& Druin, A. (2002) Family calendar survey. Retrieved September 17, 2007, from hcil .cs.umd.edu/trs/2002-21/2002-21.pdf.

Lewejohann, L., Skryabin, B. V., Sachser, N., Prehn, C., HeiDuschKa, P., Thanos, S., ET AL. (2004). Role of a neuronal small non-messenger RNA: Behavioural alterations in BC1 RNA-deleted mice. Behavioural Brain Research, 154, 273-289.

VAN DEN HoOFF, B. (2004). Electronic coordination and collective action: Use and effects of electronic calendaring and scheduling. Information \& Management, 42, 103-114.

(Manuscript received July 26, 2007; revision accepted for publication September 14, 2007.) 